\title{
CAPÍTULO 14: PURIFICAÇÃO DE PROTEASES DE Aspergillus heteromorphus URM 0269 POR SISTEMA DE DUAS FASES AQUOSAS PEG-Citrato
}

\section{CAPÍTULO 14: PURIFICACIÓN DE PROTEASAS DE Aspergillus heteromorphus URM 0269 MEDIANTE SISTEMA DE DOS FASES ACUOSAS PEG-Citrato}

\section{CHAPTER 14: PURIFICATION OF PROTEASES OF Aspergillus heteromorphus URM 0269 BY AQUEOUS TWO-PHASE SYSTEM PEG-Citrate}

\author{
Maria Eduarda Menezes Rocha ${ }^{1}$; Yuri Matheus Silva Amaral ${ }^{2}$; Rodrigo Lira de Oliveira ${ }^{3}$; Wanessa Braz da Silva \\ 4; Tatiana Souza Porto ${ }^{5}$
}

DOI: https://doi.org/10.31692/978-65-88970-19-5.212-230

\begin{abstract}
RESUMO
As proteases são enzimas capazes de catalisar a hidrólise de ligações peptídicas e apresentam diversas aplicações biotecnológicas, sendo uma das principais enzimas empregadas nos diferentes setores industriais, especialmente na indústria de alimentos. A produção dessas enzimas através de métodos fermentativos apresenta diversas vantagens como baixo custo, rendimento elevado e produção em larga escala, porém, a presença de impurezas nos extratos obtidos no final do processo pode ocasionar diminuição no potencial biotecnológico em termos de aplicações, sendo assim, necessário técnicas que proporcionem a viabilidade da função e estrutura das proteases. O Sistema de Duas Fases Aquosas (SDFA) é uma técnica simples, econômica e com elevando rendimento de extração. Diante disso, o SDFA PEG/Citrato foi utilizado para extrair e purificar parcialmente as proteases produzidas a partir do micro-organismo Aspergillus heteromorphus URM 0269 por fermentação submersa (FSm). Para essa finalidade, foi realizado um planejamento fatorial completo $2^{4}$ tendo como variáveis reposta $\mathrm{o}$ coeficiente de partição $(\mathrm{K})$, recuperação $(\mathrm{Y})$ e fator de purificação $(\mathrm{FP})$ e as variáveis independentes estudadas foram a massa molar de PEG $\left(\mathrm{M}_{\mathrm{PEG}}\right)$, concentração de $\mathrm{PEG}\left(\mathrm{C}_{\mathrm{PEG}}\right)$, concentração de citrato $\left(\mathrm{C}_{\text {CITRATO }}\right)$ e $\mathrm{pH}$. A protease apresentou maior preferência pela fase superior, rica em PEG, onde as variáveis $\mathrm{M}_{\mathrm{PEG}}$ e $\mathrm{C}_{\mathrm{CITRATO}}$, bem como a interação entre ambas, apresentaram efeitos significativos para todas as variáveis resposta. O ensaio $5\left(\mathrm{M}_{\mathrm{PEG}} 400 \mathrm{~g} / \mathrm{mol}, \mathrm{C}_{\mathrm{PEG}} 24 \% \mathrm{~m} / \mathrm{m}, \mathrm{C}_{\mathrm{CITRATO}} 20 \% \mathrm{~m} / \mathrm{m}\right.$, pH 6$)$ apresentou os melhores resultados K $(9,24)$, Y $(222,53 \%)$ e FP $(1,93)$. A utilização do SDFA se mostrou eficiente para extração e purificação parcial da protease obtida por Aspergillus heteromorphus URM 0269 apresentando elevadas recuperações, indicando a possibilidade de aplicação na extração de proteases com potencial de aplicação industrial, especialmente na indústria de alimentos.
\end{abstract}

Palavras-Chave: Aspergillus, Purificação, Proteases, SDFA.

\section{RESUMEN}

Las proteasas son enzimas capaces de catalizar la hidrólisis de enlaces peptídicos y tienen varias aplicaciones biotecnológicas, siendo una de las principales enzimas empleadas en diferentes sectores industriales, especialmente en la industria alimentaria. La producción de estas enzimas mediante

\footnotetext{
${ }^{1}$ Engenharia de Alimentos, Universidade Federal do Agreste de Pernambuco, eduardamenezes32@gmail.com

${ }^{2}$ Engenheiro de Alimentos, Universidade Federal Rural de Pernambuco, yuri.amaral17@gmail.com

${ }^{3}$ Bolsista de pós-doutorado, Universidade Federal do Agreste de Pernambuco, rodrigolira1@outlook.com

${ }^{4}$ Engenharia de Alimentos, Universidade Federal do Agreste de Pernambuco, wanessa.braz09@gmail.com

${ }^{5}$ Doutora em Tecnologia Bioquímico-Farmacêutica, Universidade Federal do Agreste de Pernambuco, portots@yahoo.com
} 
métodos fermentativos tiene varias ventajas como bajo costo, alto rendimiento y producción a gran escala, sin embargo, la presencia de impurezas en los extractos obtenidos al final del proceso puede provocar una disminución del potencial biotecnológico en términos de aplicaciones, por tanto, técnicas necesarias que proporcionen la viabilidad de la función y estructura de las proteasas. El sistema de dos fases acuoso (SDFA) es una técnica sencilla y económica con un alto rendimiento de extracción. Por tanto, se utilizó SDFA PEG/Citrato para extraer y purificar parcialmente las proteasas producidas a partir del microorganismo Aspergillus heteromorphus URM 0269 mediante fermentación sumergida (FSm). Para ello se realizó un diseño factorial completo $2^{4}$, con el coeficiente de partición $(\mathrm{K})$, recuperación (Y) y factor de purificación (FP) como variables, y las variables independientes estudiadas fueron la masa molar de PEG $\left(\mathrm{M}_{\mathrm{PEG}}\right)$, concentración de PEG $\left(\mathrm{C}_{\mathrm{PEG}}\right)$, concentración de citrato $\left(\mathrm{C}_{\text {CITRATO }}\right)$ y $\mathrm{pH}$. La proteasa mostró mayor preferencia por la fase superior, rica en PEG, donde las variables $\mathrm{M}_{\mathrm{PEG}}$ y $\mathrm{C}_{\mathrm{CITRATO}}$, así como la interacción entre ambas, mostraron los mayores efectos estadísticamente significativos para todas las variables de respuesta. El ensayo $5\left(\mathrm{M}_{\mathrm{PEG}} 400 \mathrm{~g} / \mathrm{mol}\right.$, $\mathrm{C}_{\mathrm{PEG}} 24 \% \mathrm{p} / \mathrm{p}, \mathrm{C}_{\text {CITRATO }} 20 \% \mathrm{p} / \mathrm{p}, \mathrm{pH}$ 6) mostró los mejores resultados $\mathrm{K}(9,24), \mathrm{Y}(222,53 \%)$ y FP $(1,93)$. El uso de SDFA demostró ser eficiente para la extracción y purificación parcial de la proteasa obtenida por Aspergillus heteromorphus URM 0269 mostrando altas recuperaciones, lo que indica la posibilidad de aplicación en la extracción de proteasas con potencial de aplicación industrial, especialmente en la industria alimentaria.

Palabras Clave: Aspergillus, Purificación, Proteasas, SDFA.

\section{ABSTRACT}

Proteases are enzymes capable of catalyzing the hydrolysis of peptide bonds and have several biotechnological applications, being one of the main enzymes used in different industrial sectors, especially in the food industry. The production of these enzymes through fermentative methods has several advantages such as low cost, high yield and large-scale production, however, the presence of impurities in the extracts obtained at the end of the process can cause a decrease in the biotechnological potential in terms of applications, thus, necessary techniques that provide the viability of the function and structure of the proteases. The Aqueous Two-Phase System (ATPS) is a simple, economical technique with high extraction yield. In view of this, the ATPS PEG/Citrate was used to extract and partially purify the proteases produced from the microorganism Aspergillus heteromorphus URM 0269 by submerged fermentation (FSm). For this purpose, a complete factorial design was carried out $2^{4}$, with the partition coefficient $(\mathrm{K})$, yield $(\mathrm{Y})$ and purification factor (FP) as variables, and the independent variables studied were the molar mass of PEG $\left(\mathrm{M}_{\mathrm{PEG}}\right)$, concentration of PEG $\left(\mathrm{C}_{\mathrm{PEG}}\right)$, citrate concentration $\left(\mathrm{C}_{\mathrm{CITRATE}}\right)$ and $\mathrm{pH}$. The protease showed greater preference for the upper phase, rich in $\mathrm{PEG}$, where the variables $\mathrm{M}_{\mathrm{PEG}}$ and $\mathrm{C}_{\mathrm{CITRATO}}$, as well as the interaction between both, showed the greatest statistically significant effects for all response variables. Assay $5\left(\mathrm{M}_{\mathrm{PEG}}\right.$ $\left.400 \mathrm{~g} / \mathrm{mol}, \mathrm{C}_{\mathrm{PEG}} 24 \% \mathrm{w} / \mathrm{w}, \mathrm{C}_{\text {CITRATE }} 20 \% \mathrm{w} / \mathrm{w}, \mathrm{pH} 6\right)$ showed the best results $\mathrm{K}(9.24), \mathrm{Y}(222.53 \%)$ and FP (1.93). The use of SDFA proved to be efficient for extraction and partial purification of the protease obtained by Aspergillus heteromorphus URM 0269 showing high recoveries, indicating the possibility of application in the extraction of proteases with potential for industrial application, especially in the food industry.

Keywords: Aspergillus, Purification, Proteases, ATPS.

\section{INTRODUÇÃO}

Proteases constituem um dos mais importantes grupos de enzimas e têm aplicações em diversos setores na indústria, como a têxtil, farmacêutica, de alimentos e detergentes (LADEIRA et al. 2012). Na indústria de alimentos apresenta diferentes aplicações, como na 
panificação proporcionando diminuição no tempo de mistura e facilitar a manipulação da massa, na produção de laticínios para modificar as propriedades funcionais das proteínas do ar

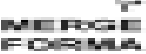
leite e reduzir o tempo global da cura do queijo, no amaciamento de carnes, assim como na diminuição da turbidez resultante das proteínas de sucos de frutas e bebidas alcoólicas, no preparo de hidrolisados de soja, de gelatina e caseína e recuperação de proteína de carne (ORLANDELLI et al. 2012; SOUZA, 2015).

A obtenção de enzimas proteolíticas, utilizando processos fermentativos apresenta certas vantagens quando em comparação a fontes animal e vegetal, pois proporciona um menor custo, altos rendimentos e produção em larga escala, porém, os extratos fermentados podem apresentar diversas outras biomoléculas como outras enzimas, ácidos orgânicos, pigmentos e compostos fenólicos (SOUZA, 2015; SOCCOL et al., 2017). O potencial biotecnológico das enzimas e consequentemente sua viabilidade econômica pode ser alterada pela presença destas impurezas, sendo necessária uma eficiente produção, atrelada as técnicas de extração e purificação capazes de preservar a estrutura e funcionalidade nativa da enzima (AMARAL et al., 2020).

Com o objetivo de garantir maior purificação de biomoléculas algumas técnicas de maior eficiência como os métodos cromatográficos são utilizadas, porém devido as grandes quantidades de contaminantes presentes nos extratos enzimáticos essas técnicas apresentam um baixo rendimento, pela desnaturação enzimática, além de serem processos com elevados custos, trabalhosos e complexos. Como alternativa o Sistemas de Duas Fases Aquosas (SDFA) atua como um processo de pré-purificação, uma vez que pode promover redução substancial de grande quantidade de contaminantes, auxiliando no aumento do rendimento em processos mais robustos de purificação (NASCIMENTO et al., 2016; PHONG et al. 2018; ABD SAMAD et al., 2017; DA SILVA et al., 2017).

Desta forma, este trabalho teve como objetivo produzir proteases de Aspergillus heteromorphus através da fermentação submersa e avaliar a extração e purificação destas enzimas através de Sistemas de Duas Fases Aquosas.

\section{FUNDAMENTAÇÃO TEÓRICA}

Proteases 
As proteases são biomoléculas capazes de catalisar a hidrólise de ligações peptídicas originando peptídeos de tamanhos variáveis ou aminoácidos livres e representam um dos principais grupos de enzimas comerciais e industriais. Estas apresentam um amplo campo de aplicações, sendo empregadas na indústria farmacêutica, cosméticos, de couro, no processamento de alimentos, na fabricação de detergentes, na produção de bebidas, entre outros (RAZZAQ et al., 2019; SANTOS, 2019).

Os microrganismos representam uma excelente fonte de proteases, sendo também a principal fonte de produção. Apesar das enzimas proteolíticas também serem obtidas de animais e plantas, os microrganismos são os principais produtores devido às suas vantagens econômicas e técnicas, além da diversidade bioquímica e da possibilidade de manipulação genética (LAXMAN et al., 2005). Essas enzimas microbianas possuem uma variedade de funções bioquímicas, fisiológicas e regulatórias. Ao longo dos séculos, as proteases microbianas desempenharam um papel fundamental na produção de alimentos fermentados tradicionais e agora o setor de enzimas industriais, dominado por produtos de protease microbiana, fornece ao mundo biocatalisadores para uso em muitas indústrias (AGUILLAR; SATO, 2018).

As proteases podem ser aplicadas em vários setores da indústria alimentícia como na produção e maturação de queijos, onde são empregadas na coagulação do leite por meio da hidrólise da ligação peptídica específica para gerar caseína e macropeptídeos (SANTOS, 2019). O amaciamento de carnes é outro processo que pode ser realizado com o uso de enzimas proteolíticas, podendo ocorrer por imersão, pulverização, adicionadas a condimentos, por injeção direta em animais vivos ou logo após o abate e também podem ser utilizadas para facilitar a separação mecânica da carne e dos ossos (DORNELLES, 2017). Na panificação, são usadas para modificar o glúten, que é uma proteína solúvel que determina as propriedades viscoelásticas e a capacidade de expansão de massa e além disso, contribuem na redução do tempo de mistura da massa e no custo da produção (AGUILAR; SATO, 2018). Além disso, as proteases podem atuar na melhoria do sabor e qualidade de hidrolisados proteicos. Geralmente, são formados a partir de caseína e proteínas do soro do leite e proteínas de soja e possuem diversas aplicações como constituintes de alimentos dietéticos e produtos naturais, formulações infantis, suplementos para nutrição clínica, além de serem usadas em alimentos para pessoas alérgicas às proteínas do leite (DORNELLES, 2017; SANTOS, 2019). 
O desenvolvimento de técnicas e métodos para a separação e purificação de enzimas tem sido um pré-requisito indispensável para muitos dos avanços feitos na indústria biotecnológica. O SDFA é bastante utilizado para purificação de biomoléculas com aplicação nos mais variados ramos da biotecnologia, tais como, enzimas, anticorpos e proteínas estruturais. Esses sistemas dividem-se em duas fases aquosas, quando são misturados dois polímeros (por exemplo, PEG e dextrana) ou um polímero e um sal apropriado (por exemplo: fosfato ou citrato) e certas condições termodinâmicas são determinadas (MARTIM et al., 2015).

Ainda assim, em muitos casos uma purificação com menor eficiência já é o suficiente, e sendo assim, o SDFA torna-se uma metodologia mais simples e econômica. Essa técnica de separação foi desenvolvida primeiramente pelo bioquímico sueco, P. A. Albertsson em 1986, e consiste na formação de duas fases entre polímeros como polietilenoglicol (PEG) e dextrana, ou entre um polímero e um sal PEG/fosfato de potássio, por exemplo, inicialmente solúveis, porém quando adicionados além das concentrações críticas de solubilidade, se tornam imiscíveis (PHONG et al. 2018). Outra característica importante desse método é a grande quantidade de água, o que garante condições não desnaturantes as biomoléculas alvo (DA SILVA et al., 2017). Essa técnica consiste no processo de purificação que engloba a integração de uma parte de extração como primeira fase de processo de retirada para sintetizar e recolher conjuntamente o bioproduto de relevância (SHOW et al., 2012).

Estudos envolvendo SDFA são fundamentais, principalmente pela incerteza em estimar o comportamento do particionamento das biomoléculas entre as fases, devido à presença de diversas interações relacionadas ao processo, como, ligações de hidrogênio, interação de cargas, força de Van der Waals, interações hidrofóbicas e efeito estérico (CHAVAN; AVHAD; RATHOD, 2015).

Sendo assim, a utilização do SDFA é uma alternativa para separação e purificação de diversos componentes biológicos e não biológicos, como proteínas recombinantes derivadas de plantas (BUYEL, TWYMAN e FISCHER, 2015), pigmentos (GÓMEZ-LOREDO, GONZÁLEZ-VALDEZ e RITO-PALOMARES, 2015), nano partículas (BENAVIDES et al., 2008) e ácidos (WANG et al., 2017), além de diversas enzimas, como lipases (RAMAKRISHNAN et al., 2016), poligalacturonase (SILVA, DE FRANÇA e PORTO, 2018), celulases (KUMAR et al., 2018), $\alpha$-amilase (SHAD et al., 2018) e proteases (AMARAL et al., 
2020). O sistema de duas fases aquosas PEG-Sal é preferido ao sistema PEG-dextrana, por apresentar maior seletividade, menor custo e um meio menos viscoso para o particionamento de proteínas, dentre os sais mais utilizados tem-se, fosfatos, sulfatos ou carbonatos de amônia, potássio ou sódio, porém o descarte de altas concentrações desses sais em águas residuais pode acarretarem problemas ao meio ambiente, como alternativa, pode se utilizar o sal citrato, que é biodegradável e atóxico (CHAVAN, AVHAD e RATHOD, 2015; DA SILVA et al., 2017; SILVA, DE FRANÇA e PORTO, 2018).

\section{METODOLOGIA}

\section{Micro-organismo}

Para a realização do trabalho foi utilizado o fungo filamentoso Aspergillus heteromorphus URM 0269 cedido pela coleção de culturas Micoteca URM da Universidade Federal de Pernambuco, mantido em tubos de ensaio inclinados contendo meio BDA (Batata Dextrose Agar) a $25 \pm 1^{\circ} \mathrm{C}$.

\section{Meio de manutenção e preparação do inóculo}

A inoculação do micro-organismo foi realizada em Erlenmeyers de $125 \mathrm{~mL}$ que continham meio de cultura BDA já esterilizados, onde foram incubados a $30^{\circ} \mathrm{C}$ em estufa por 7 dias, até que ocorresse a esporulação. Os esporos foram suspensos com a adição de solução de $\mathrm{NaCl}(0,9 \%)$ e Tween $80(0,01 \% \mathrm{v} / \mathrm{v})$ previamente esterilizados. Em seguida, foi realizada a contagem de esporos em câmara de Neubauer, até uma quantidade suficiente para concentração final $10^{5}$ esporos $/ \mathrm{mL}$, e foram inoculados nos Erlenmeyers contendo substratos para a produção de protease por fermentação submersa.

\section{Substrato para fermentação}

O substrato utilizado para fermentação submersa foi a farinha de soja, obtido do comércio local. A granulometria do substrato foi padronizada em $(<0,5 \mathrm{~mm})$ em peneiras de Tyler e em seguida seco em estufa a $65^{\circ} \mathrm{C}$ por 24 horas. Posteriormente, o substrato foi armazenamento em recipiente hermeticamente fechado à temperatura ambiente.

\section{Produção de proteases por fermentação submersa (FSm)}


A produção de proteases foi realizada em Erlenmeyers de $250 \mathrm{~mL}$ agitados a $150 \mathrm{rpm}$ e $30^{\circ} \mathrm{C}$ por $72 \mathrm{~h}$. O meio de cultura foi preparado com concentração de farinha de soja $(2 \%)$ e extrato de levedura $(0,1 \%)$. Filtrados de farinha de soja foram preparados em água destilada. O filtrado de soja foi autoclavado por 20 min a $121{ }^{\circ} \mathrm{C}$ para extração das proteínas da farinha. A mistura foi então filtrada para remover sólidos suspensos. Os sais - $0,7 \mathrm{mM}\left(\mathrm{NH}_{4}\right)_{2} \mathrm{SO}_{4}, 0,8$ $\mathrm{mM} \mathrm{MgSO}_{4} \cdot 7 \mathrm{H}_{2} \mathrm{O}$ e $5,0 \mathrm{mM} \mathrm{K} \mathrm{KPO}_{4}$ - extrato de levedura $0,5 \%$ e glicose $1 \%$ foram adicionados aos meios e foram esterilizados em autoclave sob as mesmas condições acima descritas. Após a preparação do meio de soja, foi seguida a inoculação de $10^{5}$ esporos $/ \mathrm{mL}$. Ao término da fermentação, foi realizada a filtração do extrato enzimático bruto em bomba à vácuo e as amostras foram mantidas congeladas a $-20^{\circ} \mathrm{C}$ para posterior análises.

\section{Determinação de atividade proteásica}

Para determinação da atividade proteásica foi utilizado o método descrito por Ginther (1979) em que o substrato utilizado foi a Azocaseína (1\%) com $\mathrm{CaCl}_{2} \mathrm{mM}$. A reação consistiu na adição de $0,15 \mathrm{~mL}$ do extrato enzimático e $0,25 \mathrm{~mL}$ do substrato Azocaseína $1 \%$. A mistura foi incubada por 1 hora sob o abrigo da luz a temperatura ambiente $25 \pm 1^{\circ} \mathrm{C}$. Em seguida, a reação foi interrompida com $1 \mathrm{~mL}$ de TCA $10 \%$. Foi realizada a centrifugação das amostras a $12000 \mathrm{rpm}$ por 20 minutos a $4^{\circ} \mathrm{C}$, o sobrenadante retirado e adicionado de $0,2 \mathrm{~mL}$ de $\mathrm{NaOH}$ 1,8 M. Uma unidade de atividade da protease representa a quantidade de enzima necessária para produzir uma variação de absorbância de 0,1 em 1 hora utilizando um comprimento de onda de 420nm. O resultado obtido foi expresso em $\mathrm{U} / \mathrm{mL}$.

\section{Quantificação de proteínas totais}

O teor de proteínas foi determinado através do método de Bradford (1976), utilizando-se albumina de soro bovino (BSA) como padrão.

\section{Purificação através do sistema de duas fases aquosas PEG-Citrato}

A extração por SDFA foi realizada em tubos cônicos graduados de $15 \mathrm{~mL}$ com forma cônica, contendo as quantidades desejadas de polietilenoglicol (PEG) e do sal citrato de sódio, foi adicionado o extrato bruto enzimático, que corresponde a $20 \%(\mathrm{~m} / \mathrm{m})$ da massa total do sistema. A água foi adicionada a um valor final de $5 \mathrm{~g}$. Após agitação por vórtex $1 \mathrm{~min}$, as fases foram separadas por decantação por $60 \mathrm{~min}$. Em seguida, os volumes das fases foram 
mensurados, alíquotas das fases foram retiradas separadamente com pipetas e analisadas para a concentração de proteína e atividade proteásica para os cálculos das variáveis respostas: coeficiente de partição (K), fator de purificação (FP) e recuperação (Y) calculadas de acordo com as Equações 1-3. Os parâmetros de extração estudados foram: massa molar do PEG, concentração do PEG, concentração do citrato e $\mathrm{pH}$, na extração da protease, utilizando um planejamento fatorial completo $2^{4}$. Os sistemas de suas fases aquosas foram preparados inicialmente através da mistura de PEG de diferentes massas molares em diferentes concentrações e sal citrato de sódio acrescida de ácido cítrico, para ajuste do $\mathrm{pH}$ em diferentes valores, a $25 \pm 1^{\circ} \mathrm{C}$, somando-se uma concentração final, a concentração de ácido cítrico representa $10 \%$ do total da concentração final como apresentado na Tabela 01 . Vale destacar que não é possível manter a amplitude entre o ponto $-1,0$ e +1 para a massa molar de PEG, uma vez que não é produzido solução com massa requerida (4200 g/mol), sendo o mais próximo o de $3350 \mathrm{~g} / \mathrm{mol}$. A análise estatística dos resultados foi realizada, com o auxílio do programa Statistica 7.0 (STATSOFT INC, 2008).

Tabela 01: Níveis dos fatores do Planejamento fatorial completo $2^{4}$ para purificação de proteases por Aspergillus heteromorphus URM 0269 por sistema de duas fases aquosas.

\begin{tabular}{lccc}
\hline \multicolumn{1}{c}{ Variável } & \multicolumn{3}{c}{ Nível } \\
\hline & $(-1)$ & $(0)$ & $(+1)$ \\
Massa Molar PEG $(\mathrm{g} / \mathrm{mol})$ & 400 & 3350 & 8000 \\
Concentração do PEG $(\% \mathrm{~m} / \mathrm{m})$ & 20 & 22 & 24 \\
Concentração de Citrato $(\% \mathrm{~m} / \mathrm{m})$ & 15 & 17,5 & 20 \\
$\mathrm{pH}$ & 6 & 7 & 8 \\
\hline
\end{tabular}

Fonte: O autor (2020).

\section{Definições das variáveis respostas}

O coeficiente de partição das proteases foi determinado como a razão entre atividade da protease $(U / m L)$ na fase superior rica em PEG $\left(A_{S}\right)$ e a fase inferior rica em sal $\left(A_{I}\right)$ : $K=\frac{A_{S}}{A_{I}}(1)$

O fator de purificação foi definido como a razão entre a atividade específica na fase superior ou inferior $\left(\mathrm{A}_{\mathrm{S}-\mathrm{I}} / \mathrm{C}_{\mathrm{S}-\mathrm{I}}\right)$ e seu valor inicial no extrato bruto antes da partição $\left(\mathrm{A}_{\mathrm{EB}} / \mathrm{C}_{\mathrm{EB}}\right)$ : 


\section{$F P=\frac{A S-I / C S-I}{A E B / C E B}$}

Onde, $\mathrm{C}_{\mathrm{S}-\mathrm{I}}$ e $\mathrm{C}_{\mathrm{EB}}$ são as concentrações totais de proteína $(\mathrm{mg} / \mathrm{mL})$ na fase superior ou inferior e no extrato bruto, respectivamente.

A recuperação foi determinada como a razão entre a atividade total na fase superior ou inferior $\left(\mathrm{A}_{\mathrm{S}-\mathrm{I}} \mathrm{V}_{\mathrm{S}-\mathrm{I}}\right)$ e a do extrato bruto $\left(\mathrm{A}_{\mathrm{EB}} \mathrm{V}_{\mathrm{EB}}\right)$ e expressa em porcentagem (\%):

$Y=\left(\frac{A S-I V S-I}{A E B V E B}\right) X 100$

Onde, $\mathrm{V}_{\mathrm{S}-\mathrm{I}}$ e $\mathrm{V}_{\mathrm{EB}}$ são os volumes da fase superior ou inferior e do extrato bruto, respectivamente.

\section{RESULTADOS E DISCUSSÃO}

A Tabela 02 apresenta a matriz do planejamento com os resultados obtidos para a extração e purificação da protease de Aspergillus heteromorphus URM 0269 produzida por fermentação submersa. Durante fermentação o micro-organismo apresentou uma produção de proteases $92,73 \mathrm{U} / \mathrm{mL}$. Os ensaios de extração 1, 3, 9 e 11 não formam fase, já que, as concentrações de PEG e citrato utilizadas estavam abaixo da curva binodal para o PEG de massa molar $400(\mathrm{~g} / \mathrm{mol})$ conforme reportado previamente por Porto et al. (2007).

Tabela 02: Planejamento experimental fatorial completo $2^{4}$ e resultados da purificação de proteases de Aspergillus heteromorphus URM 0269 por sistema de duas fases aquosas (SDFA) PEG/Citrato.

\begin{tabular}{cccccccc}
\hline ensaios & $\mathrm{M}_{\mathrm{PEG}}{ }^{\mathrm{a}}$ & $\mathrm{C}_{\mathrm{PEG}}{ }^{\mathrm{b}}$ & $\mathrm{C}_{\text {CITRATO }}{ }^{\mathrm{c}}$ & $\mathrm{pH}$ & $\mathrm{K}^{\mathrm{d}}$ & $\mathrm{Y}^{\mathrm{e}}(\%)$ & $\mathrm{FP}^{\mathrm{f}}$ \\
\hline 1 & 400 & 20 & 15 & 6 & - & - & - \\
2 & 8000 & 20 & 15 & 6 & 0,81 & 65,89 & 0,18 \\
3 & 400 & 24 & 15 & 6 & - & - & - \\
4 & 8000 & 24 & 15 & 6 & 0,84 & 62,18 & 0,73 \\
5 & 400 & 20 & 20 & 6 & 9,24 & 222,53 & 1,93 \\
6 & 8000 & 20 & 20 & 6 & 3,08 & 105,45 & 1,49 \\
7 & 400 & 24 & 20 & 6 & 4,43 & 199,83 & 1,35 \\
8 & 8000 & 24 & 20 & 6 & 2,61 & 133,42 & 0,66 \\
9 & 400 & 20 & 15 & 8 & - & - & - \\
10 & 8000 & 20 & 15 & 8 & 1,12 & 91,18 & 1,20 \\
11 & 400 & 24 & 15 & 8 & - & - & - \\
12 & 8000 & 24 & 15 & 8 & 0,52 & 59,94 & 0,79
\end{tabular}




\begin{tabular}{cccccccc}
13 & 400 & 20 & 20 & 8 & 2,51 & 136,26 & 0,99 \\
14 & 8000 & 20 & 20 & 8 & 0,74 & 65,20 & 0,99 \\
15 & 400 & 24 & 20 & 8 & 4,24 & 247,84 & 1,45 \\
16 & 8000 & 24 & 20 & 8 & 1,21 & 129,41 & 1,27 \\
$17(\mathrm{C})$ & 3350 & 22 & 17,5 & 7 & 0,94 & 105,01 & 0,95 \\
$18(\mathrm{C})$ & 3350 & 22 & 17,5 & 7 & 0,83 & 85,36 & 0,77 \\
$19(\mathrm{C})$ & 3350 & 22 & 17,5 & 7 & 0,93 & 90,52 & 0,83 \\
$20(\mathrm{C})$ & 3350 & 22 & 17,5 & 7 & 0,97 & 95,78 & 0,95 \\
\hline
\end{tabular}

-, Sistema que não forma fase. a - Massa molar do PEG. b - Concentração de PEG. c - Concentração de citrato. d - Coeficiente de partição. e - Recuperação de atividade na fase PEG. f - Fator de purificação na fase PEG.

Fonte: O Autor (2020).

A protease indicou a preferência pela fase superior rica em PEG, por apresentar o coeficiente de partição $\mathrm{K}>1$ para a grande maioria dos ensaios. Tanto os resultados apresentados na Tabela 02 para a Recuperação e Fator de purificação, quanto os efeitos estatísticos calculados para os mesmos parâmetros (Tabela 03) foram estipulados para fase superior rica em PEG, por ter apresentado resultados superiores para os parâmetros avaliados em relação a fase inferior (dados não mostrados).

Tabela 03: Efeitos estatísticos calculados para as respostas dos resultados de purificação de protease de Aspergillus heteromorphus URM 0269 por sistema de duas fases aquosa PEG/Citrato realizadas de acordo com o delineamento experimental $2^{4}$ da Tabela 02.

\begin{tabular}{lccc}
\hline Variável ou interação & $\mathrm{K}^{\mathrm{a}}$ & $\mathrm{Y}^{\mathrm{b}}(\%)$ & $\mathrm{FP}^{\mathrm{c}}$ \\
\hline$(1) \mathrm{M}_{\mathrm{PEG}}{ }^{\mathrm{d}}$ & $-36,56^{*}$ & $-2,76$ & $4,43^{*}$ \\
$(2) \mathrm{C}_{\mathrm{PEG}}{ }^{\mathrm{e}}$ & $-15,36^{*}$ & $4,35^{*}$ & $-1,47$ \\
$(3) \mathrm{C}_{\mathrm{CITRATO}}^{\mathrm{f}}$ & $104,02^{*}$ & $28,64^{*}$ & $20,65^{*}$ \\
$(4) \mathrm{pH}$ & $-44,88^{*}$ & $-1,77$ & 1,01 \\
$1 \times 2$ & $10,45^{*}$ & $-0,94$ & $-0,82$ \\
$1 \times 3$ & $-67,56^{*}$ & $-19,43^{*}$ & $-12,01^{*}$ \\
$1 \times 4$ & $13,26^{*}$ & $-0,51$ & $5,81^{*}$ \\
$2 \times 3$ & $-10,49^{*}$ & $6,44^{*}$ & $-2,29$ \\
$2 \times 4$ & $28,78^{*}$ & $4,26^{*}$ & $3,37^{*}$ \\
$3 \times 4$ & $-44,75^{*}$ & $-3,15^{*}$ & $-5,12^{*}$ \\
$1 \times 2 \times 3$ & $15,32^{*}$ & $-1,14$ & $-1,64$ \\
$1 \times 2 \times 4$ & $-26,15^{*}$ & $-3,74^{*}$ & $-2,58$ \\
$1 \times 3 \times 4$ & $13,39^{*}$ & $-0,87$ & 0,32 \\
$2 \times 3 \times 4$ & $34,09^{*}$ & $5,90^{*}$ & $8,87^{*}$ \\
\hline
\end{tabular}

a - Coeficiente de partição. b - Recuperação da atividade na fase PEG. c - Fator de purificação na fase PEG. d Massa molar do PEG. e - Concentração de PEG. f - Concentração de citrato. * Valores estatisticamente significativos (para $\mathrm{p}<0,05)$. 


\section{Efeitos no coeficiente de partição}

Todas as variáveis independentes assim como suas interações, apresentaram significância frente à variável resposta $\mathrm{K}$, coeficiente de partição. $\mathrm{O}$ efeito mais significante foi da variável independente $\mathrm{C}_{\text {CITRATO, }}$, sendo ele positivo, indicando que com o aumento dessa variável têm se os melhores resultados, de fato é possível observar essa influência, já que os ensaios 5, 7, e 15, onde a concentração de citrato foi superior $(20 \% \mathrm{~m} / \mathrm{m})$ os valores de K, estão acima dos demais ensaios. Em contrapartida outros ensaios que também apresentam a concentração superior de citrato, não resultaram em valores de $\mathrm{K}$ altos em relação aos ensaios 5, 7 e 15, isso ocorreu principalmente devido à massa molar do $\mathrm{PEG}$ e o $\mathrm{pH}$, tendo ambas as variáveis independentes apresentado um efeito estatístico negativo, indicando o aumento dos resultados a partir da diminuição dos níveis dessas variáveis.

Bem como o efeito antagônico entre a interação $\mathrm{C}_{\mathrm{CITRATO}}$ e $\mathrm{M}_{\mathrm{PEG}}(1 * 3)$ (Figura 01) e da interação $\mathrm{C}_{\text {CITRATo }}$ e $\mathrm{pH}(3 * 4)$, ou seja, o aumento de uma das variáveis e a diminuição da outra, resulta em maiores valores, neste caso, fica claro que o aumento da $\mathrm{C}_{\text {CITRATo }}$ e a diminuição da $\mathrm{M}_{\mathrm{PEG}}$ e do $\mathrm{pH}$ resultam nos maiores valores, todos esses efeitos explicam o maior valor encontrado no ensaio 5 de 9,24.

Figura 01: Diagrama de interpretação geométrica da interação entre $C_{\text {CITRATO }}$ e $M_{P E G}$ sobre coeficiente de partição $(\mathrm{K})$. 


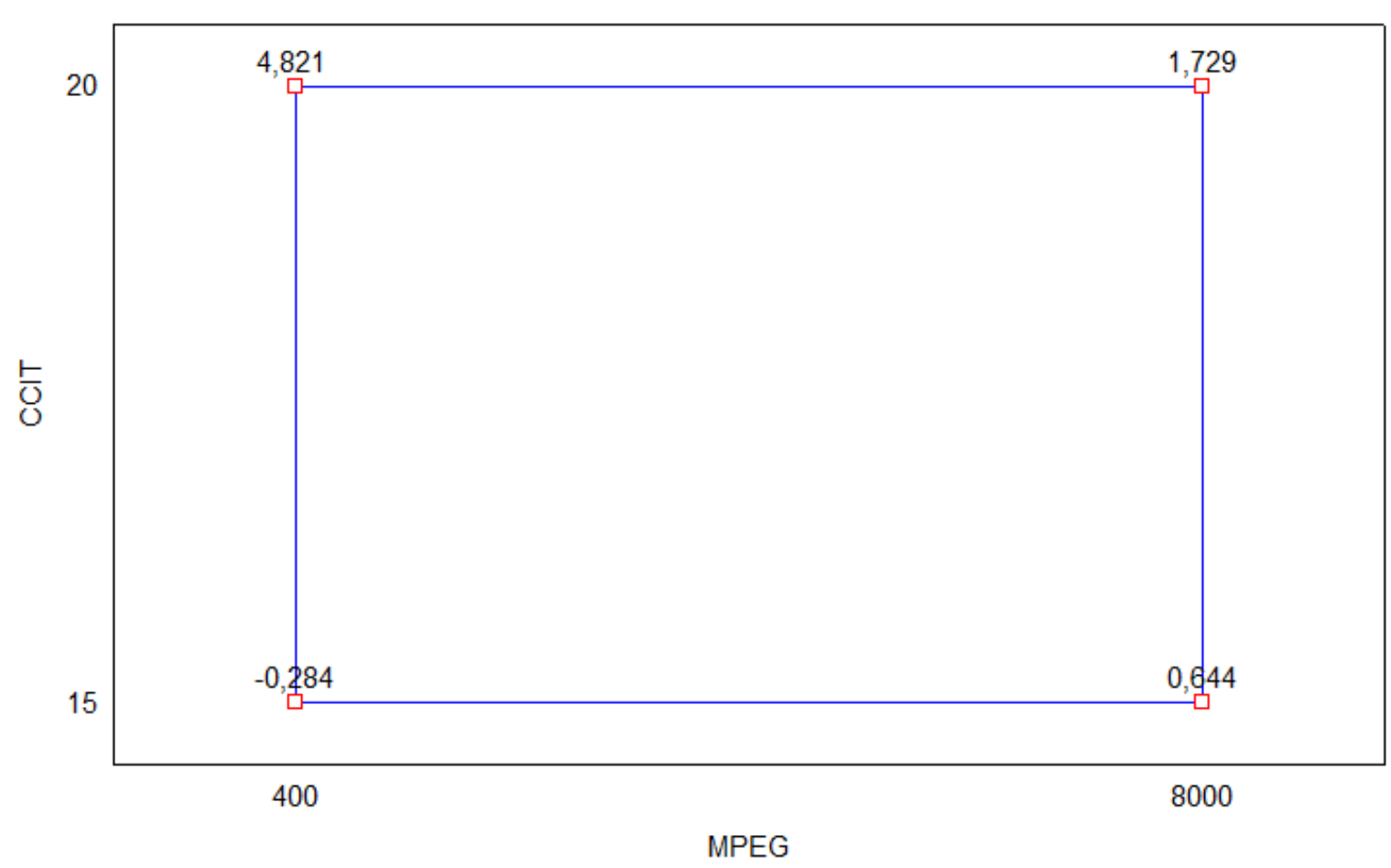

Panger $F=0$

Fonte: O autor (2020)

A razão pela tamanha importância da concentração de citrato no melhor particionamento das proteínas já é esperado, devido ao fenômeno "salting out" que comumente ocorre no caso de precipitação de proteínas, na qual grandes quantidades de sal diminuem a solubilidade das proteínas impedindo-as de se manterem na fase inferior rica em sal, e assim, direcionando-as para a fase superior rica no polímero. Por outro lado, a influência da massa molar do polímero também influencia a partição das biomoléculas, quanto menor sua massa molar do PEG, mais espaço e solvente disponível aumentando a solubilidade das proteínas (DA SILVA et al., 2017).

Outro importante efeito, foi o do $\mathrm{pH}$ que apresentou efeito significativo negativo, levando a acreditar que a enzima pode ter caráter mais alcalino, com superfície carregada positivamente no $\mathrm{pH}$ 6,0, a formação de carga pode ter contribuído para o deslocamento para a fase superior, devido justamente à intensificação de interações eletrostáticas entre o PEG e a enzima (HERCULANO et al., 2012). Neste trabalho, a protease de Aspergillus heteromorphus apresentou uma faixa de pH ótimo entre pH 7 e 8 (dados não mostrados), o que confirma a teoria de carga de superfície positiva.

Um estudo realizado por Fernandes et al. (2020) para purificar proteases de 
Aspergillus heteromorphus produzidas por fermentação em estado sólido apresentou pH ótimo 8, semelhante ao apresentado no presente trabalho. Semelhantemente os autores também observaram a partição preferencial da protease para fase PEG e observaram efeito positivo da concentração de citrato e negativo da massa molar do PEG para o K. Porém a massa molar do PEG maior $(8000 \mathrm{~g} / \mathrm{mol})$ foi melhor para purificação da enzima.

O efeito positivo da concentração de sal em resposta de maiores valores do coeficiente de partição na purificação de enzimas por sistema de duas fases aquosas também já foi relatado em diversos estudos (AMARAL et al., 2020; SILVA, DE FRANÇA e PORTO, 2018; NASCIMENTO et al., 2016; DA SILVA et al., 2017; LIMA et al., 2013). Amaral et al., (2020) durante purificação de protease de Aspergillus tamarii Kita UCP 1279 obteve efeito negativo para a interação concentração de citrato e massa molar de PEG, semelhantemente aos resultados obtidos no presente trabalho, porém verificou efeito estatístico positivo para o $\mathrm{pH}$, demonstrando as diferenças de particionamento para diferentes proteases a depender de sua carga elétrica em torno da estrutura proteica.

\section{Efeitos na Recuperação da Atividade da enzima}

Assim como o coeficiente de partição, a recuperação da atividade (Y) da protease teve efeito significativo positivo para concentração do citrato, e para a interação entre $\mathrm{C}_{\text {CITRATO }} \mathrm{e}$ $\mathrm{M}_{\mathrm{PEG}}\left(1^{*} 3\right)$ efeito negativo, como é possível observar pelos ensaios 5, 7 e $15(20 \% \mathrm{~m} / \mathrm{m}$ de citrato, massa molar do PEG $400 \mathrm{~g} / \mathrm{mol}$ ) que apresentaram recuperação na atividade da protease de $222,53 \%, 199,83 \%$ e $247,84 \%$ respectivamente, valores esses que demonstraram um alto aumento da atividade enzimática após a extração e remoção de possíveis inibidores. Além disso, altos valores de recuperação na atividade da enzima, quando o $\mathrm{K}>1$, indica que as biomoléculas não foram degradadas durante o processo, desta forma valores de Y maiores que $80 \%$ tornam viável a utilização da metodologia industrialmente (BARROS et al., 2014).

No entanto diferente do coeficiente de partição, apesar da interação $1 * 3$ significativa a massa molar de PEG $\left(\mathrm{M}_{\mathrm{PEG}}\right)$ não apresentou efeito significativo, o que demonstra que a simples diminuição da massa molar do PEG não é suficiente para a melhoria dos resultados, o mesmo ocorre para o pH. Em contrapartida a concentração de PEG apresentou efeito significativo positivo, uma vez que os melhores resultado são obtidos no nível mais alto desta variável. A concentração de $\mathrm{PEG}\left(\mathrm{C}_{\mathrm{PEG}}\right)$ foi ainda uma importante variável independente quando se tratando das interações de segunda ordem e de terceira ordem, como é o caso da 


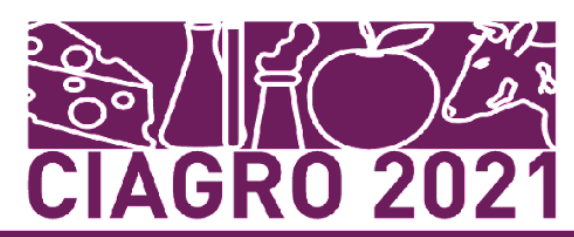

interação entre $\mathrm{C}_{\mathrm{PEG}}$ e $\mathrm{C}_{\text {CITRATO }}(2 * 3)$ (Figura 2) com efeito positivo, onde neste caso foi perceptível que o aumento das duas variáveis também promoveu um aumento em $\mathrm{Y}$, como foi nos casos dos ensaios 7 e 15 (24\% m/m de PEG e $20 \% \mathrm{~m} / \mathrm{m}$ de Citrato).

Figura 02: Diagrama de representação geométrica da interação entre $\mathrm{C}_{\mathrm{CITRATO}}$ e $\mathrm{C}_{\mathrm{PEG}}$ no rendimento $(\mathrm{Y})$ no SDFA.

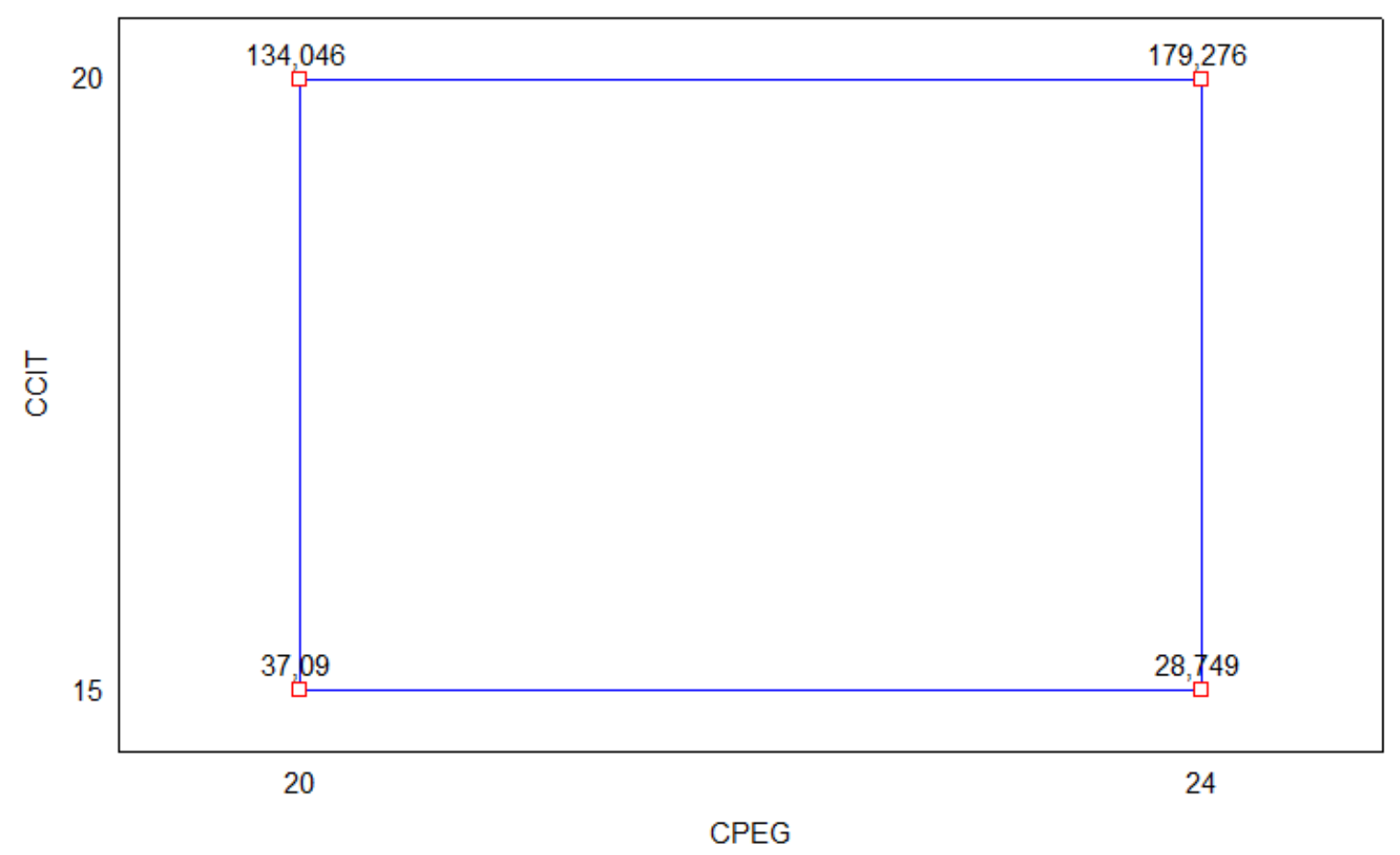

Fonte: O autor (2020).

A interação de terceira ordem entre a concentração de citrato, a concentração de PEG e o pH apresentou efeito estatístico positivo, indicando que o aumento nos níveis das três variáveis aumentou o valor da recuperação (Ensaio 15, 247,84\%). Os valores de recuperação de atividade encontrados neste trabalho e em outros artigos como Ketnawa et al., (2014) com recuperação máxima de $220 \%$ na purificação de proteases, demonstram a eficiência do SDFA em não somente apresentar baixar desnaturação enzimática, mas aumentar a atividade pela remoção de inibidores. Diferente de técnicas como a cromatografia de troca iônica que apresenta perdas por desnaturação muitas vezes acima de $80 \%$, como demonstrado por Yildirim et al., (2017).

\section{Efeitos no fator de purificação}


O fator de purificação deve ser considerado a variável mais importante no estudo da purificação de proteases de Aspergillus heteromorphus URM 0269 por SDFA, para viabilidade de aplicações industriais, pois quanto maior o fator de purificação maior a atividade especifica da protease em relação ao extrato bruto fermentado, ou seja, menor utilização de volume do extrato enzimático para o mesmo potencial catalítico.

Assim como as variáveis respostas anteriores, o fator de purificação sofreu maior

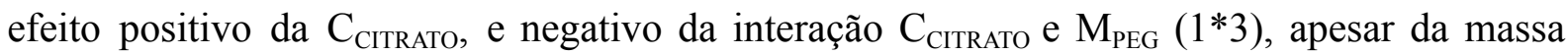
molar avaliada de forma isolada apresentar efeito positivo. De fato, a forte influência da $\mathrm{C}_{\text {CITRAto }}$ e da interação $1 * 3$ é bem evidente, dado que um aumento da concentração de $15 \%$ a $20 \%(\mathrm{~m} / \mathrm{m})$, atrelado a diferença de massa molar de PEG de 8000 para $400 \mathrm{~g} / \mathrm{mol}$ resultou em uma diferença no FP de 0,18 para 1,93 (ensaios 2 e 5 respectivamente).

Apesar da $\mathrm{C}_{\mathrm{PEG}}$ e do $\mathrm{pH}$ não apresentarem efeito estatístico significativo, a interação ternária entre $\mathrm{C}_{\mathrm{PEG}}, \mathrm{pH}$ e $\mathrm{C}_{\mathrm{CITRATO}}(2 * 3 * 4)$ foi estatisticamente significativa e apresentou interação sinérgica, indicando que o aumento das três variáveis resulta em melhoria das variáveis respostas, conforme pode ser visualizado no gráfico de representação geométrica cúbica (Figura 3), o que demonstra a importância da utilização de experimentos envolvendo planejamentos fatoriais, a fim de determinar condições de difícil observação em sistemas de purificação por SDFA.

Figura 03: Gráfico cubico dos efeitos de interação $\mathrm{C}_{\mathrm{CITRATO}}, \mathrm{C}_{\mathrm{PEG}}$, e $\mathrm{pH}$ na variável fator de purificação (FP) 


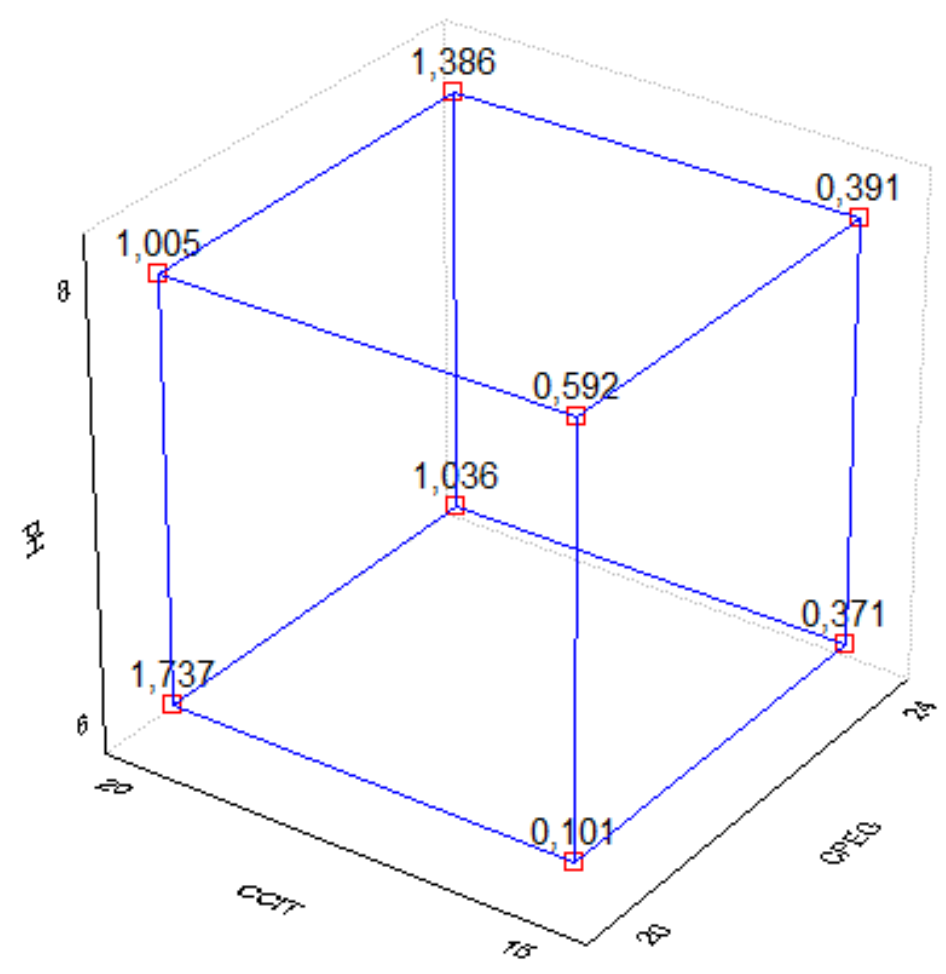

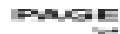
$=0$

Fonte: O autor (2020)

As variáveis respostas $\mathrm{K}$ e FP sofreram maior efeito significativo das variáveis $\mathrm{M}_{\mathrm{PEG}} \mathrm{e}$ $\mathrm{C}_{\text {CItRAto }}$ e da interação entre ambas $(1 * 3)$ e desta forma torna-se capaz a possibilidade de otimização de ambas as respostas simultaneamente, por exemplo, nas condições de $\mathrm{M}_{\mathrm{PEG}}$ $400 \mathrm{~g} / \mathrm{mol}, \mathrm{C}_{\mathrm{PEG}} 24 \% \mathrm{~m} / \mathrm{m}, \mathrm{C}_{\text {CITRATO }} 20 \% \mathrm{~m} / \mathrm{m}$, pH 6 obteve-se os melhores resultados no geral, sendo eles $\mathrm{K}=9,24, \mathrm{Y}=222,53 \%$ e $\mathrm{FP}=1,93$.

\section{CONCLUSÕES}

Os resultados obtidos no presente trabalho indicam uma extração eficiente com alta recuperação da protease produzida por Aspergillus heteromorphus URM 0269 pelo sistema de duas fases aquosas utilizando PEG/Citrato, o que promove a possibilidade de aplicação de uma metodologia simples, rápida, reprodutível e de baixo custo. A protease indicou a preferência pela fase superior rica em PEG, por apresentar o coeficiente de partição $\mathrm{K}>1$ para a grande maioria dos ensaios, sendo possível obter uma recuperação de atividade (Y) de até $247,84 \%$. De forma geral, os melhores resultados foram obtidos no ensaio 5 nas condições de $\mathrm{M}_{\text {PEG }} 400 \mathrm{~g} / \mathrm{mol}, \mathrm{C}_{\text {CITRATO }} 20 \% \mathrm{~m} / \mathrm{m}, \mathrm{C}_{\mathrm{PEG}} 20 \% \mathrm{~m} / \mathrm{m}$ e $\mathrm{pH} 6,0$, no qual $\mathrm{K}$, Y e $\mathrm{FP}$ 
corresponderam a 9,24, 222,53\% e 1,93, respectivamente. As variáveis respostas $\mathrm{K}$ e FP

sofreram maior efeito significativo da variável $\mathrm{C}_{\mathrm{CITRATO}}$ e da interação entre $\mathrm{C}_{\mathrm{CITRATO}}$ e $\mathrm{M}_{\mathrm{PEG}}$ (1*3), resultado de fenômenos como o "salting out" e maior disponibilidade de espaço e solvente, o que consequentemente promove maior solubilidade da protease pela menor massa molar do PEG. Com isso, o SDFA PEG-Citrato se apresenta como uma metodologia viável para extração de proteases que em futuros estudos poderão ser aplicadas em diversos setores, principalmente da indústria de alimentos.

\section{REFERÊNCIAS}

ABD SAMAD, N. S. A.; AMID, A.; JIMAT, D. N.; SHUKOR, N. A. A. Protease purification from Bacillus amyloliquefaciens B7 using aqueous two-phase system (ATPS). International Food Research Journal, v. 24, n. Suppl., 2017.

AGUILAR, J. G. S.; SATO, H. H. Microbial proteases: Production and application in obtaining protein hydrolysate. Food Research International, v. 103, p. 253-262, 2018.

ALBERTSSON, P. A. Partition of cell particles and macromolecules, Wiley-Interscience, New York, p.346, 1986.

AMARAL, Y. M. S.; DA SILVA, O. S.; DE OLIVEIRA, R. L.; PORTO, T. S. Production, extraction, and thermodynamics protease partitioning from Aspergillus tamarii Kita UCP1279 using PEG/sodium citrate aqueous two-phase systems. Preparative Biochemistry \& Biotechnology, 1-8, 2020.

BARROS, K. V. G.; SOUZA, P. M.; FREITAS, M. M.; FERREIRA FILHO, E. X.; JUNIOR, A. P., MAGALHÃES, P. O. PEG/NaPA aqueous two-phase systems for the purification of proteases expressed by Penicillium restrictum from Brazilian Savanna. Process Biochemistry, v. 49, n. 12, p. 2305-2312, 2014.

BENAVIDES, J.; AGUILAR, O.; LAPIZCO-ENCINAS, B. H.; RITO-PALOMARES, M. Extraction and purification of bioproducts and nanoparticles using aqueous two-phase systems strategies. Chemical Engineering \& Technology: Industrial Chemistry-Plant Equipment-Process Engineering-Biotechnology, v. 31, n. 6, p. 838-845, 2008.

BRADFORD, M.M. A rapid and sensitive method for the quantitation of microgram quantities of protein utilizing the principle of protein-dye binding. Anal. Biochem. v. 72, p. 248-254, 1976.

BUYEL, J. F.; TWYMAN, R. M.; FISCHER, R. Extraction and downstream processing of plant-derived recombinant proteins. Biotechnology advances, v. 33, n. 6, p. 902-913, 2015.

CHAVAN, R. S.; AVHAD, D. N.; RATHOD, V. K. Optimization of Aqueous Two-Phase Extraction of Protease Produced from Bacillus licheniformis NCIM 2042 Using Response Surface Methodology. Separation Science and Technology, v. 50, n. 1, p. 45-55, 2015.

DA SILVA, O. S.; GOMES, M. H. G.; DE OLIVEIRA, R. L.; PORTO, A. L. F.; CONVERTI, A.; PORTO, T. S. Partitioning and extraction protease from Aspergillus tamarii URM4634 
using PEG-citrate aqueous two-phase systems. Biocatalysis and Agricultural Biotechnology, v. 9, p. 168-173, 2017.

FERNANDES, L. M. G.; CARNEIRO-DA-CUNHA, M. N.; SILVA, J. C.; PORTO, A. L F.; PORTO, T. S. Purification and characterization of a novel Aspergillus heteromorphus URM 0269 protease extracted by aqueous two-phase systems PEG/citrate. Journal of Molecular Liquids, v. 317, 113957, 2020.

DORNELLES, L. P. Purificação, caracterização e utilização de protease de Mytella charruana (Bivalvia: Mytilidae) na obtenção de peptídeos antimicrobianos. Dissertação (mestrado em bioquímica e fisiologia) - Centro de Biociências. Bioquímica e Fisiologia, Universidade Federal de Pernambuco, 2017.

GINTHER, C. L. Sporulation and the production of serine protease and cephamycin C by Streptomyces lactamdurans. Antimicrobial Agents and Chemotherapy, 15, 522-526, 1979.

GÓMEZ-LOREDO, A.; GONZÁLEZ-VALDEZ, J.; RITO-PALOMARES, M. Insights on the downstream purification of fucoxanthin, a microalgal carotenoid, from an aqueous two-phase system stream exploiting ultrafiltration. Journal of applied phycology, v. 27, n. 4, p. 1517-1523, 2015.

HERCULANO, P. N.; PORTO, T. S.; MACIEL, M. H.; MOREIRA, K. A.; SOUZA-MOTTA, C. M.; PORTO, A. L. Partitioning and purification of the cellulolytic complex produced by Aspergillus japonicus URM5620 using PEG-citrate in an aqueous two-phase system. Fluid Phase Equilibria, v. 335, p. 8-13, 2012.

KETNAWA， S.; BENJAKUL， S.; MARTÍNEZ-ALVAREZ， O.; RAWDKUEN, S. Three-phase partitioning and proteins hydrolysis patterns of alkaline proteases derived from fish viscera. Separation and Purification Technology, 132, 174-181, 2014.

KUMAR, B.; BHARDWAJ, N.; ALAM, A.; AGRAWAL, K.; PRASAD, H.; VERMA, P. Production, purification and characterization of an acid/alkali and thermo tolerant cellulase from Schizophyllum commune NAIMCC-F-03379 and its application in hydrolysis of lignocellulosic wastes. AMB Express, v. 8, n. 1, p. 173, 2018.

LADEIRA, S. A.; DELATORRE, A. B.; ANDRADE, M. V. V.; MARTINS, M. L. L. Nota Científica: Utilização da pectina, proteínas do soro de queijo e água de maceração de milho para a produção de proteases por Bacillus sp. termofílico. Brazilian Journal of Food Technology, v. 15, n. 1, p. 92-98, 2012.

LAXMAN, R. S.; SONAWANE, A. P.; MORE, S. V.; RAO, B. S.; RELE, M. V.; JOGDAND, V. V.; DESHPANDE, V. V.; RAO, M. B. Optimization and scale up of production of alkaline protease from Conidiobolus coronatus. Process Biochemistry, v. 40, p. 3152-3158, 2005.

LIMA, C. A.; JÚNIOR, A. C. F.; LIMA FILHO, J. L.; CONVERTI, A.; MARQUES, D. A. V.; CARNEIRO-DA-CUNHA, M. G.; PORTO, A. L. F. Two-phase partitioning and partial characterization of a collagenase from Penicillium aurantiogriseum URM4622: Application to collagen hydrolysis. Biochemical engineering journal, v. 75, p. 64-71, 2013.

MARTIM, S. R.; SILVA, T. A.; TEIXEIRA, L. S.; CRUZ-FILHO, R. F.; FONSECA, T. R. B.; MARINHO, N. M. V.; SANTOS-EBINUMA, V. C.; TEIXEIRA, M. F. S. Produção e extração de proteases por fermentação extrativa. Diversidade Microbiana da Amazônia, p. 
NASCIMENTO, T. P.; SALES, A. E.; PORTO, C. S.; BRANDÃO, R. M. P.; DE CAMPOS-TAKAKI, G. M.; TEIXEIRA, J. A. C.; CONVERTI, A. Purification of a fibrinolytic protease from Mucor subtilissimus UCP 1262 by aqueous two-phase systems (PEG/sulfate). Journal of Chromatography B, v. 1025, p. 16-24, 2016.

ORLANDELLI, R. C.; SPECIAN, V.; FELBER, A. C.; PAMPHIL, J. A. Enzimas de interesse industrial: Produção por fungos e aplicações, SaBios: Rev. Saúde e Biol., v.7, n.3, p.97-109, 2012.

PHONG, W. N.; SHOW, P. L.; CHOW, Y. H.; LING, T. C. Recovery of biotechnological products using aqueous two-phase systems. Journal of bioscience and bioengineering, 2018.

RAMAKRISHNAN, V.; GOVEAS, L. C.; SURALIKERIMATH, N.; JAMPANI, C.; HALAMI, P. M.; NARAYAN, B. Extraction and purification of lipase from Enterococcus faecium MTCC5695 by PEG/phosphate aqueous-two phase system (ATPS) and its biochemical characterization. Biocatalysis and agricultural biotechnology, v. 6, p. 19-27, 2016.

RAZZAQ, A.; SHAMSI, S.; ALI, A.; ALI, Q.; SAJJAD, M.; MALIK, A.; ASHRAF, M. Microbial Proteases Applications. Frontiers in Bioengineering and Biotechnology, v. 7, 2019.

SANTOS, G. L. Produção, purificação e caracterização parcial de proteases de penicillium citrinum. Dissertação (mestrado em engenharia química) - Faculdade de Engenharia Química, Universidade Estadual de Campinas, 2019.

SHAD, Z.; MIRHOSSEINI, H.; HUSSIN, A. S. M.; FORGHANI, B.; MOTSHAKERI, M.; MANAP, M. Y. A. Aqueous two-phase purification of $\alpha$-Amylase from white pitaya (Hylocereus undatus) peel in polyethylene glycol/citrate system: Optimization by response surface methodology. Biocatalysis and agricultural biotechnology, v. 14, p. 305-313, 2018.

SHOW, P. L.; TAN, C. P.; SHAMSULANUAR M.; ARIFF, A.; YUSOF, Y. A.; CHEN, S. K.; LING, T. C.; Extractive fermentation for improved production and recovery of lipase derived from Burkholderia cepacia using a thermoseparating polymer in aqueous two-phase systems. BioresourTechnol , p. 116:226-233, 2012.

SILVA, J. C.; DE FRANÇA, P. R. L.; PORTO, T. S. Optimized extraction of polygalacturonase from Aspergillus aculeatus URM4953 by aqueous two-phase systems PEG/Citrate. Journal of Molecular Liquids, v. 263, p. 81-88, 2018.

SOCCOL, C. R.; DA COSTA, E. S. F.; LETTI, L. A. J.; KARP, S. G.; WOICIECHOWSKI, A. L.; DE SOUZA VANDENBERGHE, L. P. Recent developments and innovations in solid state fermentation. Biotechnology Research and Innovation, v. 1, n. 1, p. 52-71, 2017.

SOUZA, P. M. Produção de Proteases por Fungos Filamentosos Isolados do Cerrado do Centro-oeste Brasileiro. Tese (Doutorado) - USP, 2015. 
STASOFT, I. STATISTICA (Data Analysis Software Systems) Version 8.0, 2008.

WANG, T.; XU, W. J.; WANG, S. X.; KOU, P.; WANG, P.; WANG, X. Q.; FU, Y. J. meare Integrated and sustainable separation of chlorogenic acid from blueberry leaves by deep eutectic solvents coupled with aqueous two-phase system. Food and bioproducts processing, v. 105, p. 205-214, 2017.

YILDIRIM, V.; BALTACI, M. O.; OZGENCLI, I.; SISECIOGLU, M.; ADIGUZEL, A.; ADIGUZEL, G. Purification and biochemical characterization of a novel thermostable serine alkaline protease from Aeribacillus pallidus C10: a potential additive for detergents. Journal of enzyme inhibition and medicinal chemistry, 32(1), 468-477, 2017. 